Research Journal of Applied Sciences, Engineering and Technology 6(8): 1490-1496, 2013

DOI:10.19026/rjaset.6.3978

ISSN: 2040-7459; e-ISSN: 2040-7467

(C) 2013 Maxwell Scientific Publication Corp.

$\begin{array}{lll}\text { Submitted: October 31, } 2012 & \text { Accepted: December 28, } 2012 & \text { Published: July 10, } 2013\end{array}$

\title{
Research Article Decay of Temperature Fluctuations in Dusty Fluid Homogeneous Turbulence Prior to The Final Period
}

\author{
M.A.K. Azad and Mst. Mumtahinah \\ Department of Applied Mathematics, University of Rajshahi, Rajshahi-6205, Bangladesh
}

\begin{abstract}
Using Deissler's method we have studied the decay of temperature fluctuations in dusty fluid homogeneous turbulence before the final period and have considered correlations between fluctuating quantities at two- and three- point. The equations for two and three point correlation in presence of dust particles is obtained and the set of equations is made to determinate by neglecting the forth order correlation in comparison to the second and third order correlations. For solving the correlation equations are converted to spectral form by taking their Fourier transform. Finally integrating the energy spectrum over all wave numbers, the energy decay law of temperature fluctuations in homogeneous turbulence before the final period in presence of dust particle is obtained.
\end{abstract}

Keywords: Dust particle, Homogeneous turbulence, temperature fluctuations

\section{INTRODUCTION}

Interest in motion of dusty viscous fluid has developed rapidly in recent years. Such situations occur in movement of dust-laden air, in problems of fluidization, in the use of dust in gas cooling system and in sedimentation problem in tidal rivers.

Taylor (1935) has been pointed out that the equation of motion of turbulence relates the pressure gradient and the acceleration of the fluid particles and the mean-square acceleration can be determined from the observation of the diffusion of marked fluid particles. The behavior of dust particles in a turbulent flow depends on the concentration of the particles and the size of the particles with respect to the scale of turbulent fluid. Saffman (1962) derived an equation that describes the motion of a fluid containing small dust particle, which is applicable to laminar flows as well as turbulent flow. Kishore and Sarker (1990) studied the rate of change of vorticity covariance in MHD turbulent flow of dusty incompressible fluid. Also Rahman (2010) studied the Rate of change of vorticity covariance in MHD turbulent flow of dusty fluid in a rotating system. Kishore and Sinha (1988) also studied the rate of change of vorticity covariance of dusty fluid turbulence. Corrsin (1951b) had made an analytical attack on the problem of turbulent temperature fluctuations using the approaches employed in the statistical theory of turbulence. His results pertain to the final period of decay and for the case of appreciable convective effects, to the "energy" spectral from in specific wave- number ranges.

Deissler $(1958,1960)$ developed a theory for homogeneous turbulence, which was valid for times before the final period. Following Deissler's theory
Loeffler et al. (1961) studied the decay of temperature fluctuations in homogeneous turbulence before the final period. Sarker and Azad (2006), Azad and Sarker (2006, 2008, 2009) and Azad et al. (2006), also studied the decay of temperature fluctuations in homogeneous and MHD dusty fluid turbulence. Azad et al. (2012) studied transport equatoin for the joint distribution function of velocity, temperature and concentration in convective tubulent flow in presence of dust particles. Bkar et al. (2012) considered first-order reactant in homogeneou dusty fluid turbulence prior to the ultimate phase of decay for four-point correlation in a rotating system. Molla et al. (2012) studied the decay of temperature fluctuation in homogeneous turbulenc before the final period in a Rotating System. Sarker et al. (2012) measured Homogeneous dusty fluid turbulence in a first order reactant for the case of multi Point and multi time prior to the final period of decay.

They had considered dust particles and Coriolis force in their won works. In their study, they considered two- and three -point correlations and neglecting fourth- and higher-order correlation terms compared to the second- and third-order correlation terms. Sinha (1988) had considered the effect of dust particles on the acceleration of ordinary turbulence. Kishore and Singh (1984) had studied the statistical theory of decay process of homogeneous hydro- magnetic turbulence. Dixit and Upadhyay (1989a) also had deliberated the effect of coriolis force on acceleration covariance in MHD turbulent dusty flow with rotational symmetry. Kishore and Golsefied (1988) considered the effect of Coriolis force on acceleration covariance in MHD turbulent flow of a dusty incompressible fluid. They had also considered dust particle in their won study. 
In the present study, by analyzing the above theories we have studied the decay of temperature fluctuations in homogeneous turbulence prior to the final period in presence of dust particle considering the correlations between fluctuating quantities at two- and three- point and single time. In solving the problem, it seems logical to use the approach which has already been employed with success for studying turbulence. In this study, Deissler's method is used to solving the problem. Through the study we have obtained the energy decay law of temperature fluctuations in homogeneous dusty fluid turbulence prior to the final period. In this result, it is shown that the energy decays more rapidly than clean fluid.

\section{CORRELATION AND SPECTRAL EQUATIONS}

For an incompressible fluid with constant properties and for negligible frictional heating, the energy equation may be written at the point $\mathrm{P}$ :

$$
\left[\frac{\partial \widetilde{T}}{\partial t}+\widetilde{u}_{i} \frac{\partial \widetilde{T}}{\partial x_{i}}\right]=\frac{k}{\rho_{p}} \frac{\partial^{2} \widetilde{T}}{\partial x_{i} \partial_{i}}
$$

where,

$\tilde{T}=$ Instantaneous values of temperature

$\tilde{u}_{i}=$ Instantaneous velocity

$\rho=$ Fluid density

$\mathrm{C}_{\mathrm{p}}=$ Heat capacity at constant pressure

$\mathrm{k}=$ Thermal conductivity

$\mathrm{x}_{\mathrm{i}}=$ Space co-ordinate

$\mathrm{t}=$ Time

Separate these instantaneous values into time average and fluctuating components as $\widetilde{T}=\bar{T}+T$ and $\tilde{u}_{i}=\bar{u}_{i}+u_{i}$ Eq. (1) may be written

$$
\left[\frac{\partial \bar{T}}{\partial t}+\frac{\partial T}{\partial t}+\bar{u}_{i} \frac{\partial \bar{T}}{\partial x_{i}}+\bar{u}_{i} \frac{\partial T}{\partial x_{i}}+u_{i} \frac{\partial \bar{T}}{\partial x_{i}}+u_{i} \frac{\partial T}{\partial x_{i}}\right]=\gamma\left[\frac{\partial^{2} \bar{T}}{\partial x_{i} \partial x_{i}}+\frac{\partial^{2} T}{\partial x_{i} \partial_{i}}\right]
$$

where,

$$
\gamma=\frac{k}{\rho c_{p}}
$$

From the case of homogeneity it follows that $\frac{\partial \bar{T}}{\partial x_{i}}=0$ and in addition the usual assumption is made that $\bar{T}$ is independent of time and that $\bar{u}_{i}=0$; Thus Eq. (2) simplifies to:

$$
\left[\frac{\partial T}{\partial t}+u_{i} \frac{\partial T}{\partial x_{i}}\right]=\frac{v}{\mathrm{P}_{r}}\left[\frac{\partial^{2} T}{\partial x_{i} \partial x_{i}}\right]
$$

where, $\mathrm{P}_{r}=\frac{v}{\gamma}$, Prandtl number, $v=$ Kinematic Viscosity.
Eq. (3) holds at the arbitrary point P. For the point $\mathrm{P}$ the corresponding equation can be written:

$$
\left[\frac{\partial T^{\prime}}{\partial t}+u_{i}^{\prime} \frac{\partial T^{\prime}}{\partial x_{i}^{\prime}}\right]=\frac{v}{\mathrm{P}_{r}}\left[\frac{\partial^{2} T^{\prime}}{\partial x_{i}^{\prime} \partial x_{i}^{\prime}}\right]
$$

Multiplying Eq. (3) by T, Eq. (4) by $\mathrm{T}$ and taking time average and adding the two equations gives

$$
\left[\frac{\partial \overline{T T^{\prime}}}{\partial t}+u_{i} \frac{\partial \overline{T T^{\prime}}}{\partial x_{i}}+u_{i}^{\prime} \frac{\partial \overline{T T^{\prime}}}{\partial x_{i}^{\prime}}\right]=\frac{v}{\mathrm{P}_{r}}\left[\frac{\partial^{2} \overline{T T^{\prime}}}{\partial x_{i} \partial x_{i}}+\frac{\partial^{2} \overline{T T^{\prime}}}{\partial x_{i}^{\prime} \partial x_{i}^{\prime}}\right]
$$

The continuity equation is:

$$
\frac{\partial u_{i}}{\partial x_{i}}=\frac{\partial u_{i}^{\prime}}{\partial x_{i}^{\prime}}=0
$$

Substitution of Eq. (6) into (5) yields:

$$
\frac{\partial \overline{T T^{\prime}}}{\partial t}+u_{i} \frac{\partial \overline{u_{i} T T^{\prime}}}{\partial x_{i}}+\frac{\partial \overline{u_{i}^{\prime} T T^{\prime}}}{\partial x_{i}^{\prime}}=\frac{v}{\mathrm{P}_{r}}\left[\frac{\partial^{2} \overline{T T^{\prime}}}{\partial x_{i} \partial x_{i}}+\frac{\partial^{2} \overline{T T^{\prime}}}{\partial x_{i}^{\prime} \partial x_{i}^{\prime}}\right]
$$

By use of a new independent variable:

$$
\begin{aligned}
& r_{i}=x_{i}^{\prime}-x_{i} \text { i.e., } \\
& \frac{\partial}{\partial x_{i}}=-\frac{\partial}{\partial r_{i}}, \quad \frac{\partial}{\partial x_{i}^{\prime}}=\frac{\partial}{\partial r_{i}} \\
& \frac{\partial \overline{T T^{\prime}}}{\partial t}-\frac{\partial \overline{u_{i} T T^{\prime}}}{\partial r_{i}}+\frac{\partial \overline{u_{i}^{\prime} T T^{\prime}}}{\partial r_{i}}=\frac{2 v}{\mathrm{P}_{r}} \frac{\partial^{2} \overline{T T^{\prime}}}{\partial r_{i} \partial r_{i}}
\end{aligned}
$$

This equation is converted into spectral form by use of the following three dimensional Fourier transforms:

$$
\overline{T T^{\prime}}(\hat{r})=\int_{-\infty}^{\infty} \overline{\tau \tau^{\prime}}(\hat{K}) \exp [i(\hat{K} \cdot \hat{r})] d \hat{K}
$$

$\overline{u_{i}^{\prime} T T^{\prime}}(\hat{r})=\int_{-\infty}^{\infty} \overline{\phi_{i} \tau \tau^{\prime}}(\hat{K}) \exp [i(\hat{K} . \hat{r})] d \hat{K}$

And by interchanging $\mathrm{P}$ and $\mathrm{p}$ :

$$
\begin{aligned}
& \overline{u_{i}^{\prime} T T^{\prime}}(\hat{r})=\overline{u_{i} T T^{\prime}}(-\hat{r}) \\
& \overline{u_{i} T T^{\prime}}(\hat{r})=\int_{-\infty}^{\infty} \phi_{i} \tau \tau^{\prime}(-\hat{K}) \exp [i(\hat{K} \cdot \hat{r})] d \hat{K} \\
& \overline{u_{i} T^{\prime}}(\hat{r})=\int_{-\infty}^{\infty} \overline{\phi_{i} \tau^{\prime}}(-\hat{K}) \exp [i(\hat{K} \cdot \hat{r})] d \hat{K} \\
& \overline{u_{i}^{\prime} T}(\hat{r})=\int_{-\infty}^{\infty} \overline{\phi_{i} \tau}(\hat{K}) \exp [i(\hat{K} \cdot \hat{r})] d \hat{K}
\end{aligned}
$$


Substitution of Eq. (9-11b) into Eq. (8) leads to the spectral equation:

$\frac{\partial \overline{\tau \tau^{\prime}}(\hat{K})}{\partial t}+i k_{i}\left[\overline{\phi_{i} \tau \tau^{\prime}}(-\hat{K})-\overline{\phi_{i} \tau \tau}(\hat{K})\right]=-\frac{2 v}{\mathrm{P}_{r}} k^{2} \overline{\tau \tau^{\prime}}(\hat{K})$

Equation (12) is analogous to the two point spectral equation governing the decay of velocity fluctuations and therefore the quantity $\tau \tau^{\prime}(\mathrm{k})$ may be interpreted as a temperature fluctuation "energy" contribution of thermal eddies of size 1/k. Eq. (12) expresses the time derivative of this "energy" as a function of the convective transfer to other wave numbers and the "dissipation" due to the action of thermal conductivity. The second term on the left hand side of Eq. (12) is the so called transfer to term while the term on the right hand side is "dissipation" term.

Three points correlation and spectral equations: In order to obtain single time and three point correlation and spectral equation we consider three points $\mathrm{P}, \mathrm{P}^{\prime}$ and $\mathrm{P}^{\prime \prime}$ with position vectors $\hat{r}$ and $\hat{r}^{\prime}$ are considered.

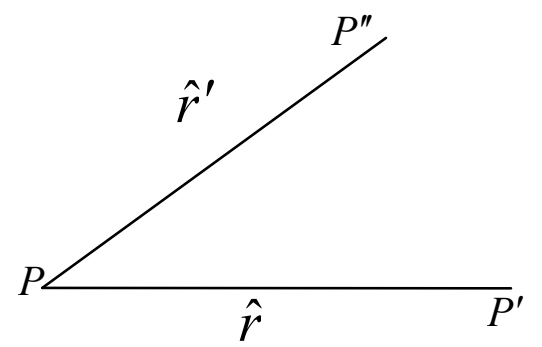

For the two points $\mathrm{P}^{\prime}$ and $\mathrm{P}^{\prime \prime}$ we can write a relation according to Eq. (7):

$\frac{\partial\left(T^{\prime} T^{\prime \prime}\right)}{\partial t}+u_{i} \frac{\partial\left(u_{i}^{\prime} T^{\prime} T^{\prime \prime}\right)}{\partial x_{i}^{\prime}}+\frac{\partial\left(u_{i}^{\prime \prime} T^{\prime} T^{\prime \prime}\right)}{\partial x_{i}^{\prime \prime}}=\frac{v}{\mathrm{P}_{r}}\left[\frac{\partial^{2}\left(T^{\prime} T^{\prime \prime}\right)}{\partial x_{i}^{\prime} \partial x_{i}^{\prime}}+\frac{\partial^{2}\left(T^{\prime} T^{\prime \prime}\right)}{\partial x_{i}^{\prime \prime} \partial x_{i}^{\prime \prime}}\right]$

Eq. (13) multiplied through by $u$, the $j$-th velocity fluctuation component at point. Then the equation can be written in a rotating system at the point $\mathrm{P}$ :

$\frac{\partial\left(u_{j} T^{\prime} T^{\prime \prime}\right)}{\partial t}+u_{i} \frac{\partial\left(u_{j} u_{i}^{\prime} T^{\prime \prime} T^{\prime \prime}\right)}{\partial x_{i}^{\prime}}+\frac{\partial\left(u_{j} u_{i}^{\prime \prime} T^{\prime} T^{\prime \prime}\right)}{\partial x_{i}^{\prime \prime}}=\frac{\nu}{\mathrm{P}_{r}}\left[\frac{\partial^{2}\left(u_{j} T^{\prime} T^{\prime \prime}\right)}{\partial x_{i}^{\prime} \partial x_{i}^{\prime}}+\frac{\partial^{2}\left(u_{j} T^{\prime} T^{\prime \prime}\right)}{\partial x_{i}^{\prime \prime} \partial x_{i}^{\prime \prime}}\right]+T^{\prime} T^{\prime \prime} \frac{\partial u_{j}}{\partial t}$

The momentum equation at point $\mathrm{P}$, in presence of dust particles:

$$
\begin{aligned}
& \frac{\partial u_{j}}{\partial t}+\frac{\partial\left(u_{j} u_{i}\right)}{\partial x_{i}}=-\frac{1}{\rho} \frac{\partial \mathrm{P}}{\partial x_{j}}+v \frac{\partial^{2} u_{j}}{\partial x_{i} \partial x_{i}}+f\left(u_{j}-v_{j}\right) \\
& \Rightarrow \frac{\partial u_{j}}{\partial t}=-\frac{\partial\left(u_{j} u_{i}\right)}{\partial x_{i}}-\frac{1}{\rho} \frac{\partial \mathrm{P}}{\partial x_{j}}+v \frac{\partial^{2} u_{j}}{\partial x_{i} \partial x_{i}}+f\left(u_{j}-v_{j}\right)
\end{aligned}
$$

Here,

$u_{j}=$ Turbulent velocity component

$v_{j}=$ Dust velocity component

$f=\frac{k N}{\rho}$ (Dimension of frequency)

$\mathrm{N}$, constant number density of dust particle

Substituted Eq. (15) into Eq. (14) the result on taking time averages is:

$$
\begin{aligned}
& \frac{\partial\left(\overline{u_{j} T^{\prime} T^{\prime \prime}}\right)}{\partial t}+u_{i} \frac{\partial\left(\overline{u_{j} u_{i}^{\prime} T^{\prime} T^{\prime \prime}}\right)}{\partial x_{i}^{\prime}}+\frac{\partial\left(\overline{u_{j} u_{i}^{\prime \prime} T^{\prime \prime} T^{\prime}}\right)}{\partial x_{i}^{\prime \prime}}=\frac{v}{\mathrm{P}_{r}}\left[\frac{\partial^{2}\left(\overline{u_{j} T^{\prime} T^{\prime \prime}}\right)}{\partial x_{i}^{\prime} \partial x_{i}^{\prime}}+\frac{\partial^{2}\left(\overline{u_{j} T^{\prime} T^{\prime \prime}}\right)}{\partial x_{i}^{\prime \prime} \partial x_{i}^{\prime \prime}}\right] \\
& -\frac{\partial\left(\overline{u_{j} u_{i} T^{\prime} T^{\prime \prime}}\right)}{\partial x_{i}}-\frac{1 \partial\left(\overline{\mathrm{P} T^{\prime} T^{\prime \prime}}\right)}{\partial x_{j}}+v \frac{\partial^{2}\left(\overline{u_{j} T^{\prime} T^{\prime \prime}}\right)}{\partial x_{i} \partial x_{i}}+f\left(\overline{u_{j} T^{\prime} T^{\prime \prime}}-\overline{v_{j} T^{\prime} T^{\prime \prime}}\right)
\end{aligned}
$$

Making use of the relations $r_{i}=x_{i}^{\prime}-x_{i}$ and $r_{i}^{\prime}=x_{i}^{\prime \prime}-x_{i}^{\prime}$ allows Eq. (16) can be written as:

$$
\begin{aligned}
& \frac{\partial\left(\overline{u_{j} T^{\prime} T^{\prime \prime}}\right)}{\partial t}-\frac{v}{\mathrm{P}_{r}}\left\{\left(1+\mathrm{P}_{r}\right) \frac{\partial^{2}\left(\overline{u_{j} T^{\prime} T^{\prime \prime}}\right)}{\partial r_{i} \partial r_{i}}+2 \mathrm{P}_{r} \frac{\partial^{2}\left(\overline{\left(u_{j} T^{\prime} T^{\prime \prime}\right.}\right)}{\partial r_{i} \partial r_{i}^{\prime}}+\left(1+\mathrm{P}_{r}\right) \frac{\partial^{2}\left(\overline{u_{j} T^{\prime} T^{\prime \prime}}\right)}{\partial r_{i}^{\prime} \partial r_{i}^{\prime}}\right\}= \\
& -\frac{\partial\left(\overline{u_{j} u_{i}^{\prime} T^{\prime} T^{\prime \prime}}\right)}{\partial r_{i}}-\frac{\partial\left(\overline{u_{j} u_{i}^{\prime \prime} T^{\prime \prime} T^{\prime}}\right)}{\partial r_{i}^{\prime}}+\frac{\partial\left(\overline{u_{j} u_{i} T^{\prime} T^{\prime \prime}}\right)}{\partial r_{i}^{\prime}}+\frac{\partial\left(\overline{u_{j} u_{i} T^{\prime} T^{\prime \prime}}\right)}{\partial r_{i}} \\
& +\frac{1}{\rho} \frac{\partial\left(\overline{\mathrm{PT}^{\prime} T^{\prime \prime}}\right)}{\partial r_{j}^{\prime}}+\frac{1}{\rho} \frac{\partial\left(\overline{\mathrm{PT}^{\prime} T^{\prime \prime}}\right)}{\partial r_{j}}+f\left(\overline{u_{j} T^{\prime} T^{\prime \prime}}-\overline{v_{j} T^{\prime} T^{\prime \prime}}\right)
\end{aligned}
$$

Six-dimensional Fourier transforms for quantities this equation may be defined as:

$$
\begin{aligned}
& \overline{u_{j} T^{\prime} T^{\prime \prime}}=\int_{-\infty-\infty}^{\infty} \int_{\beta_{j}}^{\infty} \overline{\theta^{\prime} \theta^{\prime \prime}} \exp \left[i\left(\hat{K} \cdot \hat{r}+\hat{K}^{\prime} \cdot \hat{r}^{\prime}\right)\right] d \hat{K} d \hat{K} \\
& \overline{u_{j} u_{i}^{\prime} T^{\prime} T^{\prime \prime}}=\int_{-\infty-\infty}^{\infty} \int^{\infty} \overline{\beta_{j} \beta_{i}^{\prime} \theta^{\prime} \theta^{\prime \prime}} \exp \left[i\left(\hat{K} \cdot \hat{r}+\hat{K}^{\prime} \cdot \hat{r}^{\prime}\right)\right] d \hat{K} d \hat{K} \\
& \overline{\mathrm{P}^{\prime} T^{\prime \prime}}=\int_{-\infty-\infty}^{\infty} \int_{\alpha}^{\infty} \overline{\alpha \theta^{\prime} \theta^{\prime \prime}} \exp \left[i\left(\hat{K} \cdot \hat{r}+\hat{K}^{\prime} \cdot \hat{r}^{\prime}\right)\right] d \hat{K} d \hat{K} \\
& \overline{v_{j} u_{i}^{\prime \prime} T^{\prime \prime} T^{\prime}}=\int_{-\infty-\infty}^{\infty} \int_{\gamma_{j} \theta^{\prime} \theta^{\prime \prime}} \exp \left[i\left(\hat{K} \cdot \hat{r}+\hat{K}^{\prime} \cdot \hat{r}^{\prime}\right)\right] d \hat{K} d \hat{K}
\end{aligned}
$$

Interchanging the points $\mathrm{P}^{\prime}$ and $\mathrm{P}^{\prime \prime}$ shows that:

$$
\overline{u_{j} u_{i}^{\prime \prime} T^{\prime \prime} T^{\prime}}=\overline{u_{j} u_{i}^{\prime} T^{\prime} T^{\prime \prime}}=\int_{-\infty-\infty}^{\infty} \int_{\beta_{j}}^{\infty} \beta_{i}^{\prime} \theta^{\prime} \theta^{\prime \prime} \exp \left[i\left(\hat{K} \cdot \hat{r}+\hat{K}^{\prime} \cdot \hat{r}^{\prime}\right)\right] d \hat{K} d \hat{K}
$$

Using Eq. (18-20b) into Eq. (17) then the transformed equation can be written as: 


$$
\begin{aligned}
& \frac{\partial\left(\overline{\beta_{j} \theta^{\prime} \theta^{\prime \prime}}\right)}{\partial t}+\frac{v}{\mathrm{P}_{r}}\left\{\left(1+\mathrm{P}_{r}\right) k^{2}+2 \mathrm{P}_{r} k_{i} k_{i}^{\prime}+\left(1+\mathrm{P}_{r}\right) k^{2}-\frac{p_{r}}{v} f\right\} \overline{\beta_{j} \theta^{\prime} \theta^{\prime \prime}}= \\
& -i\left(k_{i}+k_{i}^{\prime}\right) \overline{\beta_{j} \beta_{i}^{\prime} \theta^{\prime} \theta^{\prime \prime}}+i\left(k_{i}^{\prime}+k_{i}\right) \\
& \overline{\beta_{j} \beta_{i} \theta^{\prime} \theta^{\prime \prime}}+\frac{1}{\rho} i\left(k_{j}+k_{j}^{\prime}\right) \overline{\alpha \theta^{\prime} \theta^{\prime \prime}}-f \overline{\gamma_{j} \theta^{\prime} \theta^{\prime \prime}}
\end{aligned}
$$

If the derivative with respect to $\mathrm{x}$ is taken of the momentum Eq. (16) for point $\mathrm{P}$ and taking time average the resulting equation is:

$$
\frac{\partial^{2}\left(\overline{u_{j} u_{i} T^{\prime} T^{\prime \prime}}\right)}{\partial x_{j} \partial x_{i}}=-\frac{1}{\rho} \frac{\partial^{2}\left(\overline{\mathrm{P} T^{\prime} T^{\prime \prime}}\right)}{\partial x_{j} \partial x_{j}}
$$

In terms of the displacement vectors $\hat{r}$ and $\hat{r}^{\prime}$ this becomes:

$$
\begin{aligned}
& {\left[\frac{\partial^{2}}{\partial r_{j}^{\prime} \partial r_{i}^{\prime}}+2 \frac{\partial^{2}}{\partial r_{j}^{\prime} \partial r_{i}}+\frac{\partial^{2}}{\partial r_{j} \partial r_{i}}\right] \overline{u_{j} u_{i} T^{\prime} T^{\prime \prime}}} \\
& =-\frac{1}{\rho}\left[\frac{\partial^{2}}{\partial r_{j}^{\prime} \partial r_{j}^{\prime}}+2 \frac{\partial^{2}}{\partial r_{j}^{\prime} \partial r_{j}}+\frac{\partial^{2}}{\partial r_{j} \partial r_{j}}\right] \overline{\mathrm{P} T^{\prime} T^{\prime \prime}}
\end{aligned}
$$

Taking the Fourier transform of Eq. (23) and then solving for $\overline{\alpha \theta^{\prime} \theta^{\prime \prime}}$ we get:

$$
\overline{\alpha \theta^{\prime} \theta^{\prime \prime}}=\frac{-\rho\left[k_{j}^{\prime} k_{i}^{\prime}+2 k_{j}^{\prime} k_{i}+k_{j} k_{i}\right]}{\left[k_{j}^{\prime} k_{j}^{\prime}+2 k_{j}^{\prime} k_{j}+k_{j} k_{j}\right]} \overline{\beta_{j} \beta_{i} \theta^{\prime} \theta^{\prime \prime}}
$$

Eq. (24) can be used to eliminate $\overline{\alpha \theta^{\prime} \theta^{\prime \prime}}$ from Eq. (21):

Solution for times before the final period: To obtain the equation for final period of decay the third-order fluctuation terms are neglected compared to the secondorder terms. Analogously, it would be anticipated that for times before but sufficiently near to the final period the fourth-order fluctuation terms should be negligible in comparison with the third-order terms. If this assumption is made then Eq. (24) shows that the term $\overline{\alpha \theta^{\prime} \theta^{\prime \prime}}$ associated with the pressure fluctuations, should also be neglected. Thus Eq. (21) simplifies to:

$\frac{\left.\partial \overline{\beta_{j} \theta \theta^{\prime \prime}}\right)}{\partial t}+\frac{v}{\mathrm{P}_{r}}\left\{\left(1+\mathrm{P}_{r}\right) k^{2}+2 \mathrm{P}_{r} k_{i} k_{i}^{\prime}+\left(1+\mathrm{P}_{r}\right) k^{\prime 2}-\frac{p_{r}}{v} f\right\} \overline{\beta_{j} \theta \theta^{\prime \prime}}=0$

where, $R \overline{\beta_{j} \theta^{\prime} \theta^{\prime \prime}}=\overline{\gamma_{j} \theta^{\prime} \theta^{\prime \prime}}$ and $1-\mathrm{R}=\mathrm{S}, \mathrm{R}$ and $\mathrm{S}$ are arbitrary constant.

Inner multiplication of Eq. (25) by $\mathrm{k}$ and integrating between $t_{0}$ and $t$ gives:

$k_{j} \overline{\beta_{j} \theta \theta^{\prime \prime}}$

$$
=\left[k_{j} \overline{\beta_{j} \theta \theta^{\prime}}\right]_{0} \exp \left\{\begin{array}{l}
-\frac{v}{\mathrm{P}_{r}} \\
{\left[\left(1+\mathrm{P}_{r}\right) k^{2}+2 \mathrm{P}_{r} k_{i} k_{i}^{\prime} \cos \xi+\left(1+\mathrm{P}_{r}\right) k^{\prime 2}-\frac{\mathrm{P}_{r}}{v} f_{s}\right]\left(t-t_{0}\right)}
\end{array}\right\}
$$

Now, letting $r^{\prime}=0$ in equation (18) and comparing the result with the equation (10) shows that

$$
k_{i} \overline{\phi_{i} \tau \tau^{\prime}}(\hat{K})=\int_{-\infty}^{\infty} k_{i} \overline{\beta_{i} \theta^{\prime} \theta^{\prime \prime}} \hat{K} \hat{K}^{\prime} d \hat{K}^{\prime}
$$

Substituting of Eq. (26) and (27) into Eq. (12), we obtain:

$$
\begin{aligned}
& \frac{\partial \overline{\tau \tau^{\prime}}(\hat{K})}{\partial t}+\frac{2 v}{\mathrm{P}_{r}} k^{2} \overline{\tau \tau^{\prime}}(\hat{K})=\int_{-\infty}^{\infty} i k_{i} \\
& {\left[\overline{\beta_{i} \theta^{\prime} \theta^{\prime \prime}}-\beta_{i} \theta^{\prime}(-\hat{K}) \theta^{\prime \prime}\left(-\hat{K}^{\prime}\right)\right]_{0}} \\
& \times \exp \left\{-\frac{v\left(t-t_{0}\right)}{\mathrm{P}_{r}}\left[\begin{array}{l}
\left(1+\mathrm{P}_{r}\right)\left(k^{2}+k^{\prime 2}\right) \\
+2 \mathrm{P}_{r} k k^{\prime} \cos \xi-\frac{\mathrm{P}_{r}}{v} f_{S}
\end{array}\right]\right\} d \hat{K}^{\prime}
\end{aligned}
$$

Now, $d \hat{K}^{\prime}\left(\equiv d k_{1}^{\prime} d k_{2}^{\prime} d k_{3}^{\prime}\right)$ can be expressed in terms of $k^{\prime}$ and $\xi$ as (Deissler, 1958):

$$
d \hat{K}^{\prime}=-2 \pi k^{\prime 2} d(\cos \xi) d k^{\prime}
$$

Substituting Eq. (29) into (28) yields:

$$
\begin{aligned}
& \frac{\partial \overline{\tau \tau^{\prime}}(\hat{K})}{\partial t}+\frac{2 v}{\mathrm{P}_{r}} k^{2} \overline{\tau \tau^{\prime}}(\hat{K})=2 \int_{-\infty}^{\infty} 2 i \pi k_{i}\left[\overline{\beta_{i} \theta \theta^{\prime}}\left(\hat{K}, \hat{K}^{\prime}\right)-\overline{\beta_{i} \theta \theta^{\prime}}\left(-\hat{K},-\hat{K}^{\prime}\right)\right]_{0} \\
& \times\left[\int_{-1}^{1} \exp \left\{-\frac{v\left(t-t_{0}\right)}{\mathrm{P}_{r}}\left[\left(1+\mathrm{P}_{r}\right)\left(k^{2}+k^{2}\right)+2 \mathrm{P}_{r} k k^{\prime} \cos \xi-\frac{\mathrm{P}_{r}}{v} f_{s}\right]\right\} d(\cos \xi)\right] d k^{\prime}
\end{aligned}
$$

In order to find the solutions completely and following Loeffler et al. (1961), we assume that:

$i k_{i}\left[\overline{\beta_{i} \theta \theta^{\prime \prime}}\left(\hat{K}, \hat{K}^{\prime}\right)-\overline{\beta_{i} \theta^{\prime} \theta^{\prime \prime}}\left(-\hat{K},-\hat{K}^{\prime}\right)\right]_{0}=-\frac{\delta_{0}}{(2 \pi)^{2}}\left(k^{2} k^{4}-k^{4} k^{2}\right)$

where $\delta_{0}$ constant depending on the initial condition. The negative sign is placed in front of $\delta_{0}$ in order to make the transfer of energy from small to large wave no. for positive value of $\delta_{0}$. Substituting Eq. (31) into Eq. (30):

$$
\begin{gathered}
\frac{\partial \overline{\tau \tau^{\prime}}(\hat{K})}{\partial t}+\frac{2 v}{\mathrm{P}_{r}} \cdot 2 \pi k^{2} \overline{\tau \tau^{\prime}}(\hat{K})=-2 \delta_{0} \int_{0}^{\infty}\left(k^{2} k^{\prime 4}-k^{4} k^{\prime 2}\right) \\
\times\left[\int_{-1}^{1} \exp \left\{-\frac{v\left(t-t_{0}\right)}{\mathrm{P}_{r}}\left[\left(1+\mathrm{P}_{r}\right)\left(k^{2}+k^{\prime 2}\right)+2 \mathrm{P}_{r} k k^{\prime} \cos \xi-\frac{\mathrm{P}_{r}}{v} f s\right]\right\} d(\cos \xi)\right] d k^{\prime}
\end{gathered}
$$

Multiplying both sides of Eq. (32) by $k^{2}$ and defining the spectral energy function:

$$
E=2 \pi k^{2} \overline{\tau \tau^{\prime}}(\hat{K})
$$


and the resulting equation is:

$$
\frac{\partial E}{\partial t}+\frac{2 v}{\mathrm{P}_{r}} k^{2} E=w
$$

where,

$$
\begin{aligned}
& w=-2 \delta_{0} \int_{0}^{\infty}\left(k^{2} k^{\prime 4}-k^{4} k^{\prime 2}\right) k^{2} k^{\prime 2} \\
& \times\left[\int_{-1}^{1} \exp \left\{-\frac{v\left(t-t_{0}\right)}{\mathrm{P}_{r}}\left[\left(1+\mathrm{P}_{r}\right)\left(k^{2}+k^{\prime 2}\right)+2 \mathrm{P}_{r} k k^{\prime} \cos \xi-\frac{\mathrm{P}_{r}}{v} f_{S}\right]\right\}\right] d k^{\prime}
\end{aligned}
$$

Integrating Eq. (33a) w.r.to $\xi$, we have:

$$
\begin{aligned}
& w=-\frac{\delta_{0}}{2 v\left(t-t_{0}\right)} \int_{0}^{\infty}\left(k^{3} k^{\prime 5}-k^{5} k^{\prime 3}\right) \\
& \times\left[\exp \left\{-\frac{v\left(t-t_{0}\right)}{\mathrm{P}_{r}}\left[\left(1+\mathrm{P}_{r}\right)\left(k^{2}+k^{\prime 2}\right)-2 \mathrm{P}_{r} k k^{\prime}-\frac{\mathrm{P}_{r}}{v} f_{s}\right]\right\}\right] \\
& d k^{\prime}+\frac{\delta_{0}}{v\left(t-t_{0}\right)} \int_{0}^{\infty}\left(k^{3} k^{\prime 5}-k^{5} k^{\prime 3}\right) \\
& \times\left[\exp \left\{-\frac{v\left(t-t_{0}\right)}{\mathrm{P}_{r}}\left[\left(1+\mathrm{P}_{r}\right)\left(k^{2}+k^{\prime 2}\right)+2 \mathrm{P}_{r} k k^{\prime}-\frac{\mathrm{P}_{r}}{v} f_{s}\right]\right\}\right] d k^{\prime}
\end{aligned}
$$

Again integrating Eq. (33b) w.r.to $k^{\prime}$ we have

$$
\begin{aligned}
w & =\frac{\delta_{0} \sqrt{\pi} \mathrm{P}_{r}^{\frac{5}{2}}}{2 v^{\frac{3}{2}}\left(t-t_{0}\right)^{\frac{3}{2}}\left(1+\mathrm{P}_{r}\right)} \exp \left\{\frac{\mathrm{P}_{r}}{v} f s\left(t-t_{0}\right)\right\} \\
& \times \exp \left\{\frac{-k v\left(1+2 \mathrm{P}_{r}\right)\left(t-t_{0}\right)}{\mathrm{P}_{r}\left(1+\mathrm{P}_{r}\right)}\right\} \\
& \times\left\{\frac{1 \mathrm{P}_{r} k^{4}}{4 v^{2}\left(t-t_{0}\right)^{2}\left(1+\mathrm{P}_{r}\right)}+\left[\frac{5 \mathrm{P}_{r}^{2}}{\left(1+\mathrm{P}_{r}\right)^{2}}-\frac{3}{2}\right] \frac{k^{6}}{v\left(t-t_{0}\right)}+\left[\frac{\mathrm{P}_{r}^{3}}{\left(1+\mathrm{P}_{r}\right)^{3}}-\frac{\mathrm{P}_{r}}{\left(1+\mathrm{P}_{r}\right)}\right] k^{8}\right\}
\end{aligned}
$$

The Eq. (33) indicates that w must begin as $k^{4}$ for small $k$. The condition of $\mathrm{w}$ is fulfilled by the Eq. (34). It can be shown, using Eq. (34) that:

$$
\int_{0}^{\infty} w d k=0
$$

It was to be expected physically since $\mathrm{w}$ is a measure of the transfer of "energy" and the total energy transferred to all wave numbers must be zero.

The necessity for Eq. (35) to hold can be shown as follows if Eq. (10) is written for both $k$ and $-k$ and resulting equations differentiated with respect to $r_{i}$ and added, the result is, for:

$$
\hat{r}=0 \quad\left(\frac{\partial}{\partial r_{i}}=-\frac{\partial}{\partial x_{i}}\right)
$$

$$
-2 \frac{\partial}{\partial x_{i}} \overline{u_{i} T T}=\int_{-\infty}^{\infty} i k_{i}\left[\overline{\phi_{i} \tau \tau^{\prime}(\hat{K})}-\overline{\phi_{i} \tau \tau^{\prime}(-\hat{K})}\right] \quad d \hat{K}
$$

Since according to the Eq. (32), (33) and (12), $w \equiv 2 \pi i k^{2} k_{i}\left[\overline{\phi_{i} \tau \tau^{\prime}(-\hat{K})}-\overline{\phi_{i} \tau \tau^{\prime}(\hat{K})} \mid\right.$ so the Eq. (35) can be written as $-2 \frac{\partial}{\partial x_{i}} \overline{u_{i} \tau \tau^{\prime}}=\int_{-\infty}^{\infty} \frac{w}{2 \pi k^{2}} d k$ as $d \hat{K}=4 \pi k^{2} d k$ for $w=w(k, t)$ then the Eq. (35) becomes $\int_{0}^{\infty} w d k=\frac{\partial}{\partial x_{i}} \overline{u_{i} \tau t^{\prime}}=0$ The linear Eq. (33) can be solved for w as:

$$
E=\exp \left[-\frac{2 v k^{2}\left(t-t_{0}\right)}{\mathrm{P}_{r}}\right] \int w \exp \left[\frac{2 v k^{2}\left(t-t_{0}\right)}{\mathrm{P}_{r}}\right] d t+J(k) \exp \left[-\frac{2 v k^{2}\left(t-t_{0}\right)}{\mathrm{P}_{r}}\right]
$$

where, $J(k)$ is an arbitrary function of $\mathrm{k}$.

For large times, Corrsin (1951b) has shown the correct form of the expression for $\mathrm{E}$ to be:

$$
E=\frac{N_{0}}{\pi} k^{2} \exp \left[-\frac{2 v k^{2}\left(t-t_{0}\right)}{\mathrm{P}_{r}}\right]
$$

where $N_{0}$ is an constant which depends on the initial conditions. Using Eq. (37) to evaluate $J(k)$ in Eq. (36) yields:

$$
J(k)=\frac{N_{0} K^{2}}{\pi}
$$

Now, substituting the values of $\mathrm{w}$ and $J(k)$ as given by the Eq. (34) and (38) into Eq. (36) gives the equation.

$$
\begin{aligned}
& E(k, t)=\frac{N_{0} k^{2}}{\pi} \exp \left[-\frac{2 v k^{2}\left(t-t_{0}\right)}{\mathrm{P}_{r}}\right]+\frac{\delta_{0} \sqrt{\pi} \mathrm{P}_{r}^{\frac{5}{2}}}{2 v^{\frac{3}{2}}\left(1+\mathrm{P}_{r}\right)^{\frac{7}{2}}} \exp \left\{f s\left(t-t_{0}\right)\right\} \\
& \times \exp \left\{\frac{-k^{2} v\left(1+2 \mathrm{P}_{r}\right)\left(t-t_{0}\right)}{\mathrm{P}_{r}\left(1+\mathrm{P}_{r}\right)}\right\} \\
\times & {\left[\begin{array}{l}
\frac{3 \mathrm{P}_{r} k^{4}}{2 v^{2}\left(t-t_{0}\right)^{\frac{5}{2}}}+\frac{\mathrm{P}_{r}\left(7 \mathrm{P}_{r}-6\right) k^{6}}{3 v\left(1+\mathrm{P}_{r}\right)\left(t-t_{0}\right)^{\frac{3}{2}}} \\
\left.-\frac{4\left(3 \mathrm{P}_{r}^{2}-2 \mathrm{P}_{r}+3\right) k^{8}}{3\left(1+\mathrm{P}_{r}\right)^{2}\left(t-t_{0}\right)^{\frac{1}{2}}}+\frac{8 \sqrt{v}\left(3 \mathrm{P}_{r}^{2}-2 \mathrm{P}_{r}+3\right) k^{9} F(\eta)}{3\left(1+\mathrm{P}_{r}\right)^{\frac{5}{2}} \mathrm{P}_{r}^{\frac{1}{2}}}\right]
\end{array}\right] } \\
+ & \frac{\delta_{0} \sqrt{\pi} \mathrm{P}_{r}^{\frac{5}{2}}}{4 v^{\frac{3}{2}}\left(1+\mathrm{P}_{r}\right)^{\frac{7}{2}}} \times \exp ^{\frac{1}{2}}\left\{f\left(t-t_{0}\right)\right\} \\
\times & \exp \left\{\frac{-k^{2} v\left(1+2 \mathrm{P}_{r}\right)\left(t-t_{0}\right)}{\mathrm{P}_{r}\left(1+\mathrm{P}_{r}\right)}\right\} \\
\times & {\left[\begin{array}{l}
\frac{3 \mathrm{P}_{r} k^{4}}{2 v^{2}\left(t-t_{0}\right)^{\frac{5}{2}}}+\frac{\mathrm{P}_{r}\left(7 \mathrm{P}_{r}-6\right) k^{6}}{3 v\left(1+\mathrm{P}_{r}\right)\left(t-t_{0}\right)^{\frac{3}{2}}}-\frac{4\left(3 \mathrm{P}_{r}^{2}-2 \mathrm{P}_{r}+3\right) k^{8}}{3\left(1+\mathrm{P}_{r}\right)^{2}\left(t-t_{0}\right)^{\frac{1}{2}}} \\
+\frac{8 \sqrt{v}\left(3 \mathrm{P}_{r}^{2}-2 \mathrm{P}_{r}+3\right) k^{9} F(\eta)}{3\left(1+\mathrm{P}_{r}\right)^{\frac{5}{2}} \mathrm{P}_{r}^{\frac{1}{2}}}
\end{array}\right] }
\end{aligned}
$$


where,

$$
\begin{aligned}
& F(\eta)=e^{-\eta^{2} \int_{0}^{\eta} e^{x^{2}} d x} \\
& \eta=k \sqrt{\frac{v\left(t-t_{0}\right)}{\mathrm{P}_{r}\left(1+\mathrm{P}_{r}\right)}}
\end{aligned}
$$

Putting $\hat{r}=0$ in Eq. (9) and we use the definition of $\mathrm{E}$ given by the equation (39), the result is:

$$
\frac{\overline{T T^{\prime}}}{2}=\frac{\overline{T^{2}}}{2}=\int_{0}^{\infty} E(k) d k
$$

Substituting Eq. (39) into (42) gives:

$$
\begin{aligned}
& \frac{\bar{T}^{2}}{2}=\frac{N_{0}\left(\mathrm{P}_{r}\right)^{\frac{3}{2}}}{8 \sqrt{(2 \pi)} v^{\frac{3}{2}}\left(t-t_{0}\right)^{\frac{3}{2}}}+\frac{\delta_{0} R}{v^{6}\left(t-t_{0}\right)^{5}} \exp \left[f_{s}\right] \\
& \Rightarrow \bar{T}^{2}=A\left(t-t_{0}\right)^{-\frac{3}{2}}+B \exp [f s] \times\left(t-t_{0}\right)^{-5}
\end{aligned}
$$

where,

$$
A=\frac{N_{0}\left(\mathrm{P}_{r}\right)^{\frac{3}{2}}}{4 \sqrt{(2 \pi)} v^{\frac{3}{2}}}, \quad B=\frac{2 \delta_{0} R}{v^{6}}
$$

and

$$
\begin{aligned}
& R=\frac{\pi\left(\mathrm{P}_{r}\right)^{6}}{2\left(1+\mathrm{P}_{r}\right)\left(1+2 \mathrm{P}_{r}\right)^{\frac{5}{2}}}\left\{\frac{9}{16}+\frac{5 \mathrm{P}_{r}\left(7 \mathrm{P}_{r}-6\right)}{16\left(1+2 \mathrm{P}_{r}\right)}-\frac{35 \mathrm{P}_{r}\left(3 \mathrm{P}_{r}^{2}-2 \mathrm{P}_{r}+3\right)}{8\left(1+2 \mathrm{P}_{r}\right)^{2}}\right. \\
& \left.+\frac{1.5422 \mathrm{P}_{r}\left(3 \mathrm{P}_{r}^{2}-2 \mathrm{P}_{r}+3\right)\left(1+2 \mathrm{P}_{r}\right)^{\frac{5}{2}}}{\sqrt{(\pi)}\left(1+\mathrm{P}_{r}\right)^{\frac{11}{2}}}\left[1+\sum_{n=1}^{\infty} \frac{(11) \cdots \cdot \cdot[11+2(n-1)]}{(2 n+1) n !(2)^{2 n}\left(1+\mathrm{P}_{r}\right)^{n}}\right]\right\}
\end{aligned}
$$

$\mathrm{R}$ is a function of Prandtl no.

Eq. (43) is the decay law of temperature fluctuation in homogeneous turbulence in presence of dust particle prior to the ultimate period. The first term of the right side of Eq. (43) corresponds to the temperature energy for two-point correlation and the second terms represents the energy for the three -point correlation. This second term becomes negligible at large times leaving the final period decay law previously found by Corrsin (1951b). $\bar{T}^{2}$ is the total "energy" (the mean square of the temperature fluctuations).

\section{RESULTS AND DISCUSSION}

Loeffler et al. (1961) derived the following equation of the decay of temperature fluctuations in homogeneous turbulence before the final period:

$$
\frac{\overline{T^{2}}}{2}=\frac{\left.N_{0}\left(\mathrm{P}_{r}\right)\right)^{\frac{3}{2}} v^{-\frac{3}{2}}}{8 \sqrt{(2 \pi)\left(t-t_{0}\right)^{\frac{3}{2}}}}+\frac{\delta_{0} R}{v^{6}\left(t-t_{0}\right)^{5}}
$$

$$
=A\left(t-t_{0}\right)^{-\frac{3}{2}}+B\left(t-t_{0}\right)^{-5}
$$

Here,

$$
A=\frac{N_{0}\left(\mathrm{P}_{r}\right)^{\frac{3}{2}} v^{-\frac{3}{2}}}{8 \sqrt{(2 \pi)}} \text { and } B=\frac{\delta_{0} R}{v^{6}}
$$

In their study, they considered two- and three-point correlations and neglecting fourth- and higher-order correlation terms compared to the second- and thirdorder correlation terms.

Following Loeffler et al. (1961), Molla et al. (2012) studied the decay of temperature fluctuation in homogeneous turbulenc before the final period in a Rotating System and derivrd the following equation:

$$
\begin{aligned}
& \frac{\bar{T}^{2}}{2}=\frac{N_{0}\left(\mathrm{P}_{r}\right)^{\frac{3}{2}}}{8 \sqrt{(2 \pi)} v^{\frac{3}{2}}\left(t-t_{0}\right)^{\frac{3}{2}}}+\frac{\delta_{0} R}{v^{6}\left(t-t_{0}\right)^{5}} \exp \left[-2 \varepsilon_{m i j} \Omega_{m}\right] \\
& \Rightarrow \bar{T}^{2}=A\left(t-t_{0}\right)^{-\frac{3}{2}}+B \exp \left[-2 \varepsilon_{m i j} \Omega_{m}\right] \times\left(t-t_{0}\right)^{-5}
\end{aligned}
$$

In their study they had considered corriolis force.

In the present study, I have studied the decay of temperature fluctuations in homogeneous turbulence prior to the final period taking dust particle and considered the correlations between fluctuating quantities at two- and three- point and single time. In this study, I have used Deissler's (1958) method to solving the problem. Through the study we have obtained the following energy decay law of temperature fluctuations in homogeneous dusty fluid turbulence prior to the final period. In this result, it is shown that the energy decays more rapidly than clean fluid.

$$
\begin{aligned}
& \frac{\bar{T}^{2}}{2}=\frac{N_{0}\left(\mathrm{P}_{r}\right)^{\frac{3}{2}}}{8 \sqrt{(2 \pi)} v^{\frac{3}{2}}\left(t-t_{0}\right)^{\frac{3}{2}}}+\frac{\delta_{0} R}{v^{6}\left(t-t_{0}\right)^{5}} \exp [f s] \\
& \Rightarrow \bar{T}^{2}=A\left(t-t_{0}\right)^{-\frac{3}{2}}+B \exp [f s] \times\left(t-t_{0}\right)^{-5}
\end{aligned}
$$

In the absence of the dust particle, i.e. $f=0$, then the Eq. (47) becomes

$$
\begin{aligned}
& \frac{\overline{T^{2}}}{2}=\frac{N_{0}\left(\mathrm{P}_{r}\right) \frac{3}{2} v^{-\frac{3}{2}}}{8 \sqrt{(2 \pi)}\left(t-t_{0}\right)^{\frac{3}{2}}}+\frac{\delta_{0} R}{v^{6}\left(t-t_{0}\right)^{5}} \\
& =A\left(t-t_{0}\right)^{-\frac{3}{2}}+B\left(t-t_{0}\right)^{-5}
\end{aligned}
$$

Same as Eq. (45) which was obtained earlier by Loeffler et al. (1961).

Due to the effect of dust particle in homogeneous turbulence, the temperature energy decays more rapidly then the energy for clean fluid prior to the ultimate period. For large times, the second term in the Eq. (47) 
becomes negligible leaving the $-3 / 2$ power decay law for the ultimate period.

\section{ACKNOWLEDGMENT}

The author (M.A.K.Azad) thankfully acknowledge the Faculty of Science, Rajshahi University, Bangladesh for granting fund and are also thankful to the Department of Applied Mathematics, University of Rajshahi for providing all facilities during this study.

\section{REFERENCES}

Azad, M.A.K. and M.S.A. Sarker, 2006. Decay of temperature fluctuations in homogeneous turbulence before the final period for the case of multi-point and multi-time in presence of dust particle. Rajshahi Univ. Stud. B-J. Sci., 34: 37-50.

Azad, M.A.K. and M.S.A. Sarker, 2008. Decay of temperature fluctuations in homogeneous turbulence before the final period for the case of multi-point and multi-time in a rotating system in presence of dust particle. J. Appl. Sci. Res., 4(7): 793-802.

Azad, M.A.K., M.S.A. Sarker and N.I. Mondal, 2006. Decay of temperature fluctuations in dusty fluid MHD turbulence before the final period in a rotating system. J. Eng. Appl. Sci., 1(2): 187-194.

Azad, M.A.K. and M.S.A. Sarker, 2009. Decay of temperature fluctuations in MHD turbulence before the final period in a rotating system. Bangladesh J. Sci. Ind. Res. 44(4): 407-414.

Azad, M.A.K., M.H.U. Molla and M.Z. Rahman, 2012. Transport equatoin for the joint distribution function of velocity, temperature and concentration in convective tubulent flow in presence of dust particles. Res. J. Appl. Sci. Eng. Technol., 4(20): 4150-4159.

Bkar, P.K., M.A., M.A.K. Azad and M.S. Alam Sarker, 2012. First-order reactant in homogeneou dusty fluidturbulence prior to the ultimate phase of decay for four-point correlation in a rotatingsystem. Res. J. Math. Stat., 4(2): 30-38.

Corrsin, S., 1951b. On the spectrum of isotropic temperature fluctuations in isotropic turbulence, J. Appl. Phys., 22: 469-473.
Deissler, R.G., 1958. On the decay of homogeneous turbulence before the final period. Phys. Fluids, 1: 111-121.

Deissler, R.G., 1960. A theory of decaying homogenous turbulence. Phys. Fluids, 3: 176-187.

Dixit, T. and B.N. Upadhyay, 1989a. The effect of coriolis force on acceleration covariance in MHD turbulent dusty flow with rotational symmetry. Astrophys. Space Sci., 153: 257-268.

Kishore, N. and S.R. Singh, 1984. Statistical theory of decay process of homogeneous hydro-magnetic turbulence. Astrophys. Space Sci., 104: 121-125.

Kishore, N. and Y.T. Golsefied, 1988. Effect of coriolis force on acceleration covariance in MHD turbulent flow of a dusty incompressible fluid. Astrophys. Space Sci., 150(1): 89-101.

Kishore, N. and M.S.A. Sarker, 1990. Rate of change of vortices covariance in MHD turbulent flow of dusty incompressible fluid. I. J. Energ. Res., 14(5): 573-577.

Loeffler, A.L. and R.G. Deissler, 1961. Decay of temperature fluctuations in homogeneous turbulence before the final period. Int. J. Heat Mass Transfer, 1: 312-324.

Molla, M.H.U., M.A.K. Azad and M.Z. Rahman, 2012. Decay of temperature fluctuations in homo geneousturbulenc before the finaln period in a rotating system. Res. J. Math. Stat., 4(2): 45-51.

Rahman, M.L., 2010. Rate of vortices covariance in MHD turbulent flow of dusty fluid in a rotating system. J. Mech. Cont. Math. Sci., 5(1): 570-578.

Saffman, P.G., 1962. On the stability of laminar flow of a dusty gas. J. Fluid Mech., 13: 120-128.

Sarker, M.S.A. and M.A.K. Azad, 2006. Decay of temperature fluctuations in homogeneous turbulence before the final period for the case of multi-point and multi-time in a rotating system. Bangladesh J. Sci. Ind. Res., 41(3-4): 147-158.

Sarker, M.S.A., M.A. Bkar and M.A.K. Azad, 2012. Homogeneous dusty fluid turbulence in a first order reactant for the case of multi point and multi time prior to the final period of decay. IOSR J. Math., 3(5): 39-46.

Sinha, A., 1988. Effect of dust particles on the acceleration covariance of ordinary turbulence. J. Sci. Res., B.H.U., India, 38: 7.

Taylor, G.I., 1935. Statistical theory of turbulence. 1-Iv, Proc. Roy. Soc., London A151, 874: 421-478. 